\title{
Effect of Bio-Fertilizers and Flumioxazin on Microflora and Yield of Groundnut (Arachis hypogaea L.) in Alfisol of West Bengal
}

\author{
Manasi Sahoo, Soumi Mukhopadhyay and Pabitra Kumar Biswas* \\ Department of Soil Science and Agricultural Chemistry, Palli Siksha Bhavana \\ (Institute of Agriculture), Visva-Bharati, Sriniketan-731236, W.B., India \\ *Corresponding author
}

\section{A B S T R A C T}

\begin{tabular}{|l|}
\hline Ke y w o r d s \\
Biofertilizers, \\
$\begin{array}{l}\text { Flumioxazin, } \\
\text { Groundnut, } \\
\text { Herbicide, } \\
\text { Microflora }\end{array}$ \\
\hline Article Info \\
\hline $\begin{array}{l}\text { Accepted: } \\
\text { 04 August } 2019 \\
\text { Available Online: } \\
\text { 10 September } 2019\end{array}$ \\
\hline
\end{tabular}

Introduction

Groundnut (Arachis hypogaea L.) is one of the principal economic oilseed crops in the world. It contains about $50 \%$ oil, $25-30 \%$ protein, $20 \%$ carbohydrate and $5 \%$ fibre and besides this, it also contains vitamin E, niacin, folacin, calcium, phosphorus, magnesium, zinc, iron, riboflavin, thiamine and potassium (Savage and Keenan, 1994), which make a substantial contribution to human nutrition. The oils not only acts as the essential part of human diet but also serve as an important raw materials

\begin{abstract}
Herbicide is the most important input in the modern agriculture. The use of herbicides has been expanding more rapidly than that of other pesticides. The injudicious application of herbicides in agriculture causes the contamination of the soil with toxic chemicals and become harmful to the microorganisms, plant, wildlife and man. In view of the above a field experiment was conducted during rabi season of 2015-16 with the groundnut variety TAG-24 with twenty four treatment combination in three replications. The result of this experiment showed that the application of herbicide Flumioxazin along with different combinations of biofertilizers PSB, Rhizobium and Azotobacter have no significant adverse effects in the physico-chemical properties of soil ( $\mathrm{pH}$ and EC), available nitrogen content, microflora population (NFB, PSB, fungi, actinomycetes) and pod yield of Groundnut.
\end{abstract}

for the agro-based industries and for the manufacturing of various sophisticated products.

The new approach for farming often referred to as "sustainable agriculture" advocates the use of renewable inputs like biofertilizers, green manure, vermicompost etc. This is also important both from the view point of environmentally safe technologies and providing some sort of fertilizer to the resource-poor and marginal farmers. Seed inoculation with biofertilizer is a low cost 
input which plays a significant role in crop yields and enhances nutrient availability to the crop plants.

Among the various biotic stresses resulting in low productivity, weeds are considered as a major constraint; especially under rainfed ecosystems. Weeds cause serious problems to the groundnut crop during the first 45 days of its growth. The most critical period of weed competition is from 3-6 weeks after sowing. The average yield loss due to weeds is about $30 \%$, whereas under poor management yield loss by weeds may be $60 \%$ (Dayal et al., 1987).

Use of herbicides for weed control in legumes and especially in groundnut has certainly contributed to the increased yield and improved quality. However, detrimental effects caused by these herbicides on soil microorganisms growth and metabolism have also been reported in several studies. Experiments carried out to evaluate the effect of different herbicides on the Rhizobium growth and nitrogen fixation activity revealed that the effect depends on the herbicide, its concentration, crop, nature and type of microorganisms and different weather conditions (Sawicka and Selwet, 1998). Hence, unique combination of the above factors, which are very specific for a region, needs to be studied to predict the influence of the herbicides used on the growth and metabolism of microorganisms. With this view, the present study was taken up to study the "Effect of Biofertilizers and Flumioxazin on microflora and yield of Groundnut (Arachis hypogaea L.) in alfisol of West Bengal".

\section{Materials and Methods}

\section{Site of experiment}

A field trial was conducted during rabi season of 2015-16 in Agricultural Research Farm, Institute of Agriculture, Visva-Bharati,
Sriniketan located at $23^{\circ} 39^{\prime} \mathrm{N}$ latitude and $87^{\circ} 42^{\prime} \mathrm{E}$ longitude with an altitude $58.9 \mathrm{~m}$ AMSL. The soil of the experimental site was sandy loam in texture, acidic in reaction $(\mathrm{pH}$ $4.8)$ with low level of organic carbon $(0.42 \%)$ but medium level of available nitrogen (225.79 $\mathrm{kg} \mathrm{ha}^{-1}$ ), available $\mathrm{P}_{2} \mathrm{O}_{5}(43.68 \mathrm{~kg}$ $\left.\mathrm{ha}^{-1}\right)$, available $\mathrm{K}_{2} \mathrm{O}\left(138.7 \mathrm{~kg} \mathrm{ha}^{-1}\right)$ and contained $9.37 \times 10^{4}, 18.5 \times 10^{4}, 16 \times 10^{4}, 3 \times 10^{4}$ cfu $\mathrm{g}^{-1}$ NFB, PSB, fungi and actinomycetes, respectively. The groundnut crop variety TAG-24, a bunch type, Spanish, variety which is improved through selection and contains nearly $50 \%$ of oil, matures in 110 to 120 days if sown by the first week of February. It was sown during first week of February.

\section{Experimental details}

The experiment was carried out in a Randomized Block Design with factorial concept (FRBD) having two factor. Factor A having 3 treatments i.e. No herbicide (Ho), Recommended dose of herbicide $\left(\mathrm{H}_{1}\right)$ and Double dose of herbicide $\left(\mathrm{H}_{2}\right)$ and Factor B having 7 treatments i.e. Noinoculation $\left(\mathrm{B}_{0}\right)$,PSB ( $\left.\mathrm{B}_{1}\right)$, Rhizobium (Rhizo) $\left(\mathrm{B}_{2}\right),\left(\mathrm{B}_{3}\right)$, Azotobacter (Azo) $\left(\mathrm{B}_{4}\right)$, $\mathrm{PSB}+$ Rhizobium $\left(\mathrm{B}_{5}\right)$, PSB+ Azotobacter $\left(\mathrm{B}_{6}\right)$, Rhizobium + Azotobacter $\quad\left(\mathrm{B}_{7}\right), \quad \mathrm{PSB}+\quad$ Rhizobium + Azotobacter $\left(\mathrm{B}_{8}\right)$. The interaction between two factor $\mathrm{A} \times \mathrm{B}$ gives 24 treatments which is replicated thrice. Each plot was $3 \mathrm{~m} \mathrm{x} 4 \mathrm{~m}$ surrounded by ridges. Adequate number of irrigation channels was constructed to provide irrigation independently to each plot.

\section{Sampling and analysis}

Ten plants were randomly selected from each plot and the number of pods from those plants, number of grains per pod and harvest index was determined after harvest. Soil samples were collect from the experimental plot after final harvest of the crop. Then the soil samples were dried in shade and processed in the laboratory and finally the individual test for 
soil $\mathrm{pH}$ and EC, available nitrogen content and microbial population were estimated accordingly.

\section{Results and Discussion}

\section{Effect of biofertilizers and flumioxazin on pod yield of groundnut}

The result showed that the single inoculation of Azotobacter @ $80 \mathrm{~g} \mathrm{~kg}^{-1}$ of seed without any application of herbicide gave the remarkably highest pod yield $\left(3416.67 \mathrm{~kg} \mathrm{ha}^{-}\right.$ ${ }^{1}$ ) after harvest of groundnut crop in alfisol of West Bengal followed by single inoculation of Rhizobium along with double the recommended dose of herbicide @ $500 \mathrm{~g} \mathrm{ha}^{-1}$ (3175 kg ha ${ }^{-1}$ ) and then followed by single inoculation of Rhizobium along with recommended dose of herbicide @ $250 \mathrm{~g} \mathrm{ha}^{-1}$ $\left(3091.67 \mathrm{~kg} \mathrm{ha}^{-1}\right)$. The pod yield advantages due to single inoculation of Azotobacter, single inoculation of Rhizobium, dual inoculation of Rhizobium + Azotobacter, and dual inoculation of Rhizobium + PSB were 28.39, 24.05, 14.96 and $1.54 \%$, respectively, over uninoculated control $\left(2172.11 \mathrm{~kg} \mathrm{ha}^{-1}\right)$, $32.80,28.31,18.91$ and $5.03 \%$, respectively, over dual inoculation of Azotobacter+ PSB (2099.99kg ha $\left.\mathrm{kg}^{-1}\right), 42.61,37.78,27.69$ and $12.78 \%$, respectively, over combined inoculation of Rhizobium + Azotobacter + PSB $\left(1955.55 \mathrm{~kg} \mathrm{ha}^{-1}\right)$ and 67.33, 61.67, 49.83 and $32.33 \%$, respectively, over single inoculation of PSB (1666.66 $\mathrm{kg} \mathrm{ha}^{-1}$ ) at harvest of the crop.

Single inoculation of seeds with Azotobacter significantly increased the mean pod yield $\left(2788.88 \mathrm{~kg} \mathrm{ha}^{-1}\right)$ in groundnut. This was followed by seed inoculation with Rhizobium alone (2694.44 kg ha-1) and dual inoculation of Rhizobium + Azotobacter (2497.11 $\mathrm{kg} \mathrm{ha}^{-1}$ ), irrespective of herbicide application. The yield increments might be due to improvement in number of different microbial as well as total microbial population, improvement of favourable soil physico-chemical properties and increase of available nitrogen. Shashidhar et al., (2009), Narula et al., (2000) reported significantly higher yield and total microbial population due seed inoculation with biofertilizers and their different combinations along with recommended dose herbicide (Table 1).

Effect of biofertilizers and flumioxazin on phsico-chemical properties of groundnut cropped soil

\section{Effect on soil pH and EC}

The result showed that either in the single seed inoculation of Rhizobium, PSB and Azotobacter or the different combinations of Rhizobium, PSB and Azotobacter inoculation combined with different levels of herbicide $\mathrm{H}_{0}, \mathrm{H}_{1}, \mathrm{H}_{2}$ gave more or less same results of soil $\mathrm{pH}$ after harvest of groundnut. There was no significant difference found among the treatments.

The result showed that single inoculation of Azotobacter@80 $\mathrm{g} \mathrm{kg}^{-1}$ seeds along with double RD of herbicide @ $500 \mathrm{~g} \mathrm{ha}^{-1}$ increased the EC of soil after harvest of groundnut crop in alfisol of West Bengal followed by combined inoculation of $\mathrm{PSB}+$ Rhizo+ Azotobacter along with double RD of herbicide and PSB+ Rhizo+ Azotobacter along with RD of herbicide and then followed by uninoculated control (Table $2)$. These results corroborated with the earlier findings of Sumathi et al., (2012).

\section{Effect on available nitrogen content}

The effect of herbicide and seed inoculation on available nitrogen content was found significant (Table 3). Interaction effect of Azotobacter @ $80 \mathrm{~g} \mathrm{~kg}^{-1}$ seed along with double recommended dose of herbicide @ 500 $\mathrm{g}$ ha ${ }^{-1}$ gives significantly higher nitrogen 
content $\left(561.97 \mathrm{~kg} \quad \mathrm{ha}^{-1}\right)$ followed by combined inoculation of PSB + Rhizobium +Azotobacter @ $80 \mathrm{~g} \mathrm{~kg}^{-1}$ seed without application of any herbicide (549.43 kg ha $\left.{ }^{-1}\right)$ and Single inoculation of Rhizobium @ $80 \mathrm{~g}$ $\mathrm{kg}^{-1}$ seed along with recommended dose of herbicide@ $250 \mathrm{~g} \mathrm{ha}^{-1}$ and Single inoculation of Azotobacter@80 $\mathrm{g} \mathrm{kg}^{-1}$ seed along with recommended dose of herbicide @ $250 \mathrm{~g} \mathrm{ha}^{-1}$ $\left(524.34 \mathrm{~kg} \mathrm{ha}^{-1}\right)$ in respect of all the treatments of herbicides irrespective of doses.

Based on these results obtained from the study it could, thus, be concluded that seed inoculation with different strains of biofertilizers along with herbicide may be an effective recommendation for better nitrogen management in groundnut plot. These results corroborated with the earlier findings of Usha et al., (2004) in Kinnow mandarin.

\section{Effect of biofertilizers and flumioxazin on microbial population of groundnut cropped} soil

\section{Effect on bacteria in soil}

The result showed that combined inoculation of Rhizobium + Azotobacter + PSB@80 g $\mathrm{kg}^{-1}$ of seeds along without application of herbicide gave significantly the highest number of Nitrogen fixing bacterial (NFB) at 60 DAS over initial NFB population $(9.37 \mathrm{x}$ $10^{4} \mathrm{cfu} \mathrm{g}^{-1}$ ) (Table 4) and the dual inoculation of Rhizobium + Azotobacter @ $80 \mathrm{~g} \mathrm{~kg}^{-1}$ of seeds along without application of herbicide gave the highest number of Phosphate solubilizing bacterial (PSB) population at 60 DAS over initial PSB population (18.5 x $10^{4}$ $\mathrm{cfu} \mathrm{g}^{-1}$ ) in groundnut field in alfisol of West Bengal (Table 5).

These results were in agreement with the earlier findings of Kunc et al., (1985), Taiwo and Oso (1997) in soil in respect to increase of NFB and PSB population.

\section{Effect on fungi population in soil}

The effect of interaction between herbicide and seed inoculation on fungal population was not found significant (Table 6) particularly at 15 DAS and 90 DAS onward of groundnut. The highest number of fungal population (46.50 $\times 10^{4} \mathrm{cfu}^{-1}$ ) was recorded in the treatment of PSB @ $80 \mathrm{~g} \mathrm{~kg}^{-1}$ of seed inoculation without any herbicide application at 30 DAS as compared to uninoculated control (31.52x $\left.10^{4} \mathrm{cfu} \mathrm{g}^{-1}\right)$ followed by dual inoculation of PSB + Azotobacter @ $80 \mathrm{~g} \mathrm{~kg}^{-1}$ seed along with recommended dose of herbicide @ 250g ha ${ }^{-1}\left(42.9 \times 10^{4} \mathrm{cfu} \mathrm{g}^{-1}\right)$. Based on these results obtained from the study it could, thus, be concluded that seed inoculation with different strains of biofertilizers with or without recommended dose of herbicide would be an effective recommendation for better crop management in groundnut in respect of better decomposition of organic matter as well as fertilizers due to increase of fungal population is soil. These results were in agreement with the earlier findings of Kunc et al., (1985) and Taiwo and Oso (1997) in soil in respect to increase of fungal population.

\section{Effect on actinomycetes population in soil}

The results showed that single inoculation of Azotobacter along with recommended dose of Flumioxzin@250 g ha ${ }^{-1}$ (Fig. 1) gave the highest number of Actinomycetes population at 30 DAS of groundnut in alfisol of West Bengal followed by without any application of biofertilizers along with recommended dose of herbicide@250 $\mathrm{g} \mathrm{ha}^{-1}$ at 60 DAS and without any application of biofertilizers along with double recommended dose of herbicide @ $500 \mathrm{~g} \mathrm{ha}^{-1}$ at 30 DAS (Table 7). Based on these results obtained from the study it could, thus, be concluded that seed inoculation with different strains of biofertilizers with recommended dose of herbicide would be an 
effective recommendation for better crop management in groundnut in respect of better decomposition of organic matter as well as fertilizers due to increase of actinomycetes population is soil. These results were in agreement with the earlier findings of Kunc et al., (1985) and Taiwo and Oso (1997) in soil in respect to increase of actinomycetes population.

\section{Effect on total microflora in soil}

The results showed that dual inoculation of Rhizobium + Azotobacter gave significantly highest number of microflora at 60DAS of groundnut in the alfisol of West Bengal without application of herbicide followed by dual inoculation of biofertilizer of Rhizobium + Azotobacter along with double the recommended dose of Flumioxzin @ $500 \mathrm{~g}$ $\mathrm{ha}^{-1}$ and dual inoculation of Rhizobium +PSB along with recommended dose of Flumioxazin @ $250 \mathrm{~g} \mathrm{ha}^{-1}$ (Table 8). These results were in agreement with the earlier findings of Kunc et al., (1985) and Taiwo and Oso (1997) in soil in respect to increase of total microbial population.

Table.1 Effect of biofertilizers and flumioxazin on pod yield of groundnut

\begin{tabular}{|c|c|c|c|c|}
\hline \multicolumn{5}{|c|}{ Yield $\left(\mathrm{kg} \mathrm{ha}^{-1}\right)$} \\
\hline \multirow{2}{*}{$\begin{array}{c}\text { Treatments } \\
\text { Biofert/ } \\
\text { Herbicide }\end{array}$} & \multicolumn{4}{|c|}{ *HARVEST } \\
\hline & $\mathrm{H}_{0}$ & $\mathrm{H}_{1}$ & $\mathrm{H}_{2}$ & Mean \\
\hline Control & 2441.33 & 1633.33 & 2441.67 & 2172.11 \\
\hline PSB & 958.33 & 2741.67 & 1300.00 & 1666.66 \\
\hline Rhizo & 1816.67 & 3091.67 & 3175.00 & 2694.44 \\
\hline Azo & 3416.67 & 2216.67 & 2733.33 & 2788.88 \\
\hline PSB+Rhizo & 2191.67 & 2675.00 & 1750.00 & 2205.55 \\
\hline PSB+Azo & 1933.33 & 1841.67 & 2525.00 & 2099.99 \\
\hline Azo+Rhizo & 2408.22 & 2350.00 & 2733.11 & 2497.11 \\
\hline PSB+Azo+Rhizo & 1450.00 & 1366.67 & 3050.00 & 1955.55 \\
\hline Mean & 2077.03 & 2239.58 & 2463.51 & \\
\hline $\operatorname{S.Em}( \pm)$ & \multicolumn{4}{|c|}{110634.76} \\
\hline CD $5 \%$ for $\mathrm{H}$ & \multicolumn{4}{|c|}{193.20} \\
\hline B & \multicolumn{4}{|c|}{315.62} \\
\hline HB & \multicolumn{4}{|c|}{546.60} \\
\hline CV \% & \multicolumn{4}{|c|}{14.72} \\
\hline
\end{tabular}

*Average of the three replication 
Table.2 Effect of biofertilizers and flumioxazin on $\mathrm{pH}$ and $\mathrm{EC}$ of soil after harvest of groundnut

\begin{tabular}{|c|c|c|c|c|c|c|c|c|c|c|}
\hline \multirow{3}{*}{$\begin{array}{l}\text { Treatments } \\
\text { Biofert/Herbicide }\end{array}$} & \multicolumn{5}{|c|}{ Soil pH } & \multicolumn{5}{|c|}{ Soil EC $\left(\mathrm{msm}^{-1}\right)$} \\
\hline & *Initial & \multicolumn{4}{|c|}{ *HARVEST } & *Initial & \multicolumn{4}{|c|}{ *HARVEST } \\
\hline & \multirow[t]{10}{*}{4.5} & $\mathrm{H}_{0}$ & $\mathrm{H}_{1}$ & $\mathrm{H}_{2}$ & Mean & \multirow[t]{10}{*}{1.0} & $\mathrm{H}_{0}$ & $\mathrm{H}_{1}$ & $\mathrm{H}_{2}$ & Mean \\
\hline Control & & 4.53 & 4.45 & 4.53 & 4.50 & & 1.37 & 0.92 & 1.07 & 1.12 \\
\hline PSB & & 4.50 & 4.41 & 4.75 & 4.55 & & 1.19 & 1.08 & 0.97 & 1.08 \\
\hline Rhizo & & 4.37 & 4.64 & 4.44 & 4.48 & & 0.88 & 1.06 & 0.96 & 0.97 \\
\hline Azo & & 4.53 & 4.48 & 4.35 & 4.45 & & 1.35 & 1.31 & 1.93 & 1.53 \\
\hline PSB+Rhizo & & 4.79 & 4.38 & 4.48 & 4.54 & & 0.89 & 0.95 & 1.25 & 1.03 \\
\hline PSB+Azo & & 4.47 & 4.41 & 4.43 & 4.43 & & 0.70 & 1.30 & 0.65 & 0.88 \\
\hline Azo+Rhizo & & 4.47 & 4.51 & 4.46 & 4.48 & & 1.02 & 1.28 & 0.92 & 1.07 \\
\hline PSB+Azo+Rhizo & & 4.43 & 4.38 & 4.37 & 4.39 & & 1.16 & 1.55 & 1.55 & 1.42 \\
\hline Mean & & 4.51 & 4.45 & 4.47 & & & 1.07 & 1.18 & 1.16 & \\
\hline S. $\operatorname{Em}( \pm)$ & \multicolumn{4}{|c|}{0.213} & & \multicolumn{4}{|c|}{0.02} & \\
\hline CD $5 \%$ for $\mathrm{H}$ & \multicolumn{4}{|c|}{ NS } & & \multicolumn{4}{|c|}{0.082} & \\
\hline B & \multicolumn{4}{|c|}{ NS } & & \multicolumn{4}{|c|}{0.134} & \\
\hline HB & \multicolumn{4}{|c|}{ NS } & & \multicolumn{4}{|c|}{0.232} & \\
\hline $\mathrm{CV} \%$ & \multicolumn{4}{|c|}{10.30} & & \multicolumn{4}{|c|}{12.42} & \\
\hline
\end{tabular}

*Average of the three replication

Table.3 Effect of biofertilizers and flumioxazin on available soil nitrogen

\begin{tabular}{|c|c|c|c|c|c|}
\hline \multirow{3}{*}{$\begin{array}{l}\text { Treatments } \\
\text { Biofert/Herbici } \\
\text { de }\end{array}$} & \multicolumn{5}{|c|}{ Available Nitrogen $\left(\mathrm{kg} \mathrm{ha}^{-1}\right)$ [Average of the three replication] } \\
\hline & \multirow{10}{*}{$\begin{array}{l}\text { Initial } \\
225.79\end{array}$} & \multicolumn{4}{|c|}{ Harvest } \\
\hline & & $\mathrm{H}_{0}$ & $\mathrm{H}_{1}$ & $\mathrm{H}_{2}$ & Mean \\
\hline Control & & 402.24 & 436.53 & 423.99 & 420.92 \\
\hline PSB & & 486.71 & 373.81 & 336.18 & 398.90 \\
\hline Rhizo & & 436.53 & 524.34 & 476.16 & 478.34 \\
\hline Azo & & 411.44 & 524.34 & 561.97 & 499.25 \\
\hline PSB+Rhizo & & 411.44 & 361.27 & 411.44 & 394.72 \\
\hline PSB+Azo & & 348.72 & 386.36 & 386.36 & 373.81 \\
\hline Azo+Rhizo & & 411.44 & 398.90 & 361.27 & 390.54 \\
\hline $\begin{array}{l}\text { PSB+Azo+Rhiz } \\
\text { o }\end{array}$ & & 549.43 & 449.08 & 323.64 & 440.71 \\
\hline Mean & & 432.24 & 431.82 & 409.87 & \\
\hline S. Em ( $( \pm)$ & \multicolumn{5}{|c|}{3296.38} \\
\hline CD 5\% for $\mathrm{H}$ & \multicolumn{5}{|c|}{33.36} \\
\hline B & \multicolumn{5}{|c|}{54.48} \\
\hline HB & \multicolumn{5}{|c|}{94.36} \\
\hline CV \% & \multicolumn{5}{|c|}{13.52} \\
\hline
\end{tabular}


Table.4 Effect of biofertilizers and flumioxazin on NFB population

\begin{tabular}{|c|c|c|c|c|c|c|c|c|c|c|c|c|c|c|c|c|c|c|c|c|c|}
\hline \multicolumn{22}{|c|}{ NFB Population } \\
\hline 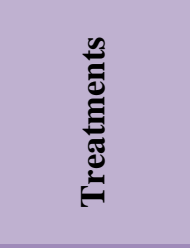 & \multirow[t]{2}{*}{ 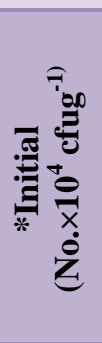 } & \multicolumn{4}{|c|}{ 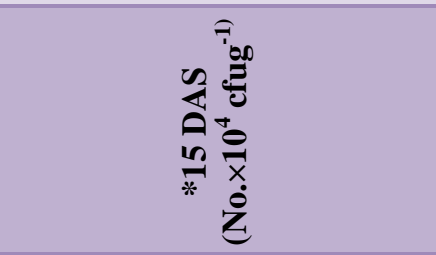 } & \multicolumn{4}{|c|}{ 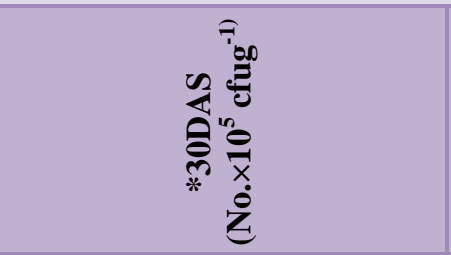 } & \multicolumn{4}{|c|}{ 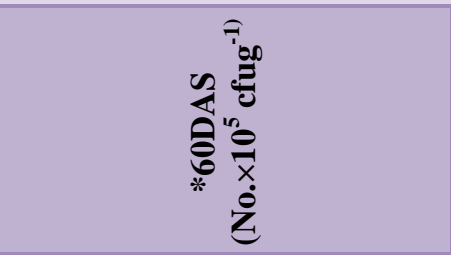 } & \multicolumn{4}{|c|}{ 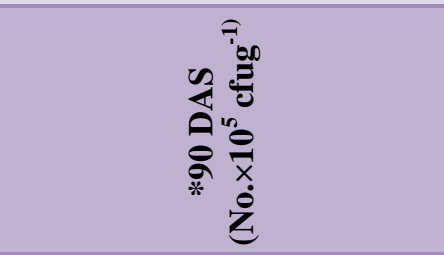 } & \multicolumn{4}{|c|}{ 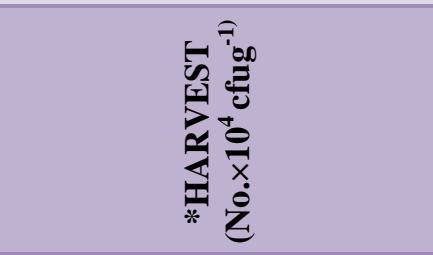 } \\
\hline $\begin{array}{c}\text { Biofert/ } \\
\text { Herbicide }\end{array}$ & & $\mathbf{H}_{\mathbf{0}}$ & $\mathbf{H}_{1}$ & $\mathbf{H}_{2}$ & Mean & $\mathbf{H}_{0}$ & $\mathbf{H}_{1}$ & $\mathbf{H}_{2}$ & Mean & $\mathbf{H}_{\mathbf{0}}$ & $\mathbf{H}_{1}$ & $\mathbf{H}_{2}$ & Mean & $\mathbf{H}_{\mathbf{0}}$ & $\mathbf{H}_{1}$ & $\mathbf{H}_{2}$ & Mean & $\mathbf{H}_{\mathbf{0}}$ & $\mathbf{H}_{1}$ & $\mathbf{H}_{2}$ & Mean \\
\hline Control & \multirow[t]{8}{*}{9.37} & 6 & 13 & 58.25 & 25.75 & 3.8 & 2.85 & 3.75 & 3.47 & 30.07 & 17.40 & 25.40 & 24.29 & 7.42 & 7.17 & 6.55 & 7.05 & 11 & 8 & 28.5 & 15.83 \\
\hline PSB & & 36.25 & 11.25 & 45.72 & 31.08 & 3.5 & 6.78 & 3.57 & 4.62 & 15.85 & 33.70 & 21.85 & 23.8 & 7.30 & 3.85 & 4.62 & 5.26 & 18.5 & 3.5 & 4.5 & 8.83 \\
\hline Rhizo & & 4 & 40.5 & 23.5 & 22.67 & 6.4 & 13.32 & 3.17 & 7.63 & 24.50 & 39.65 & 21.67 & 28.61 & 10.65 & 7.70 & 6.67 & 8.34 & 5.5 & 28.5 & 17.5 & 17.17 \\
\hline Azo & & 20.75 & 45.5 & 36.25 & 34.17 & 6.7 & 4.7 & 7.55 & 6.32 & 13.80 & 22.70 & 19.77 & 18.76 & 2.15 & 9.27 & 5.82 & 5.75 & 16 & 40 & 12 & 22.67 \\
\hline PSB+Rhizo & & 10.25 & 21.5 & 13.5 & 15.08 & 3.6 & 4.65 & 5 & 4.42 & 31.07 & 39.75 & 18.52 & 29.78 & 8.27 & 11.12 & 4.07 & 7.82 & 9 & 26.5 & 16 & 17.16 \\
\hline PSB+Azo & & 74 & 11.5 & 15.25 & 33.58 & 1.9 & 3.80 & 3.65 & 3.12 & 17.60 & 23.80 & 30.47 & 23.96 & 6.60 & 4.85 & 8.30 & 6.58 & 16 & 16 & 8.5 & 13.5 \\
\hline Azo+Rhizo & & 14.75 & 39.75 & 50 & 34.83 & 5.55 & 4.07 & 5.07 & 4.90 & 19.97 & 24.47 & 18.90 & 21.12 & 10.05 & 8.62 & 7.52 & 8.73 & 6.33 & 17 & 2 & 8.44 \\
\hline $\begin{array}{c}\text { PSB+Azo+ } \\
\text { Rhizo }\end{array}$ & & 25.25 & 56.5 & 25.75 & 35.83 & 4.525 & 4.97 & 3.92 & 4.48 & 42.58 & 23.02 & 26.10 & 30.57 & 8.97 & 7.90 & 5.45 & 7.44 & 11 & 35 & 15 & 20.33 \\
\hline Mean & & 23.91 & 29.94 & 33.53 & & 4.50 & 5.64 & 4.46 & & 24.43 & 28.06 & 22.84 & & 7.68 & 7.56 & 6.13 & & 11.67 & 21.81 & 13 & \\
\hline $\operatorname{S.Em}( \pm)$ & & \multicolumn{4}{|c|}{2.280} & \multicolumn{4}{|c|}{0.358} & \multicolumn{4}{|c|}{2.449} & \multicolumn{4}{|c|}{1.012} & \multicolumn{4}{|c|}{1.133} \\
\hline $\begin{array}{c}\text { CD 5\% for } \\
\mathrm{H}\end{array}$ & & \multicolumn{4}{|c|}{0.435} & \multicolumn{4}{|c|}{0.172} & \multicolumn{4}{|c|}{0.451} & \multicolumn{4}{|c|}{0.290} & \multicolumn{4}{|c|}{0.307} \\
\hline B & & \multicolumn{4}{|c|}{0.711} & \multicolumn{4}{|c|}{0.282} & \multicolumn{4}{|c|}{0.737} & \multicolumn{4}{|c|}{0.474} & \multicolumn{4}{|c|}{0.501} \\
\hline HB & & \multirow{2}{*}{\multicolumn{4}{|c|}{$\begin{array}{l}1.233 \\
\mathbf{5 1 8 5}\end{array}$}} & \multirow{2}{*}{\multicolumn{4}{|c|}{0.488}} & \multicolumn{4}{|c|}{1.277} & \multicolumn{4}{|c|}{0.821} & \multicolumn{4}{|c|}{0.869} \\
\hline CV \% & & & & & 5.185 & & \multicolumn{3}{|c|}{12.30} & & 6.2 & & & & 14 & & & & 6. & & \\
\hline
\end{tabular}

*Average of the three replication 
Table.5 Effect of biofertilizers and flumioxazin on PSB population

\begin{tabular}{|c|c|c|c|c|c|c|c|c|c|c|c|c|c|c|c|c|c|c|c|c|c|}
\hline \multicolumn{22}{|c|}{ PSB Population } \\
\hline 莺 & \multirow{2}{*}{ 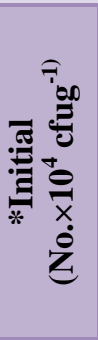 } & \multicolumn{4}{|c|}{ 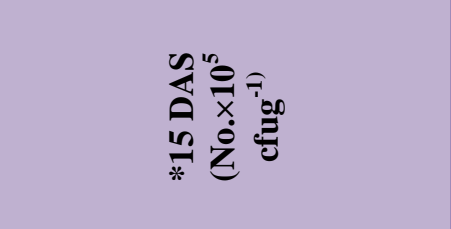 } & \multicolumn{4}{|c|}{ 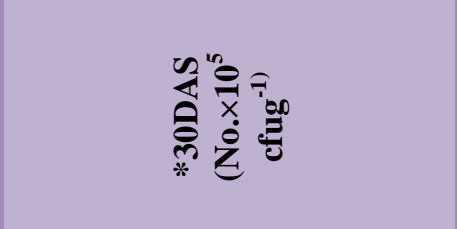 } & \multicolumn{4}{|c|}{ 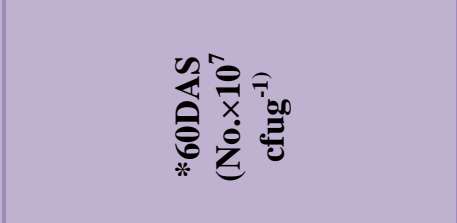 } & \multicolumn{4}{|c|}{ 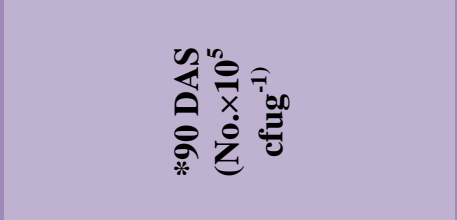 } & \multicolumn{4}{|c|}{ 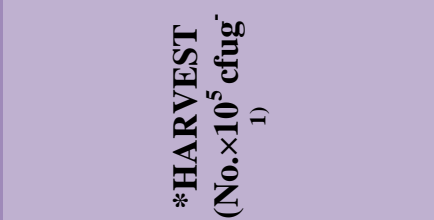 } \\
\hline $\begin{array}{l}\text { Biofert/ } \\
\text { Herbicide }\end{array}$ & & $\mathbf{H}_{\mathbf{0}}$ & $\mathbf{H}_{1}$ & $\mathbf{H}_{2}$ & Mean & $\mathbf{H}_{\mathbf{0}}$ & $\mathbf{H}_{1}$ & $\mathbf{H}_{2}$ & Mean & $\mathbf{H}_{\mathbf{0}}$ & $\mathbf{H}_{1}$ & $\mathbf{H}_{2}$ & Mean & $\mathbf{H}_{\mathbf{0}}$ & $\mathbf{H}_{1}$ & $\mathbf{H}_{2}$ & Mean & $\mathbf{H}_{\mathbf{0}}$ & $\mathbf{H}_{1}$ & $\mathbf{H}_{2}$ & Mean \\
\hline Control & \multirow[t]{8}{*}{18.5} & 7.22 & 5.85 & 2.63 & 5.24 & 12 & 15.83 & 28.31 & 18.71 & 3.62 & 18.67 & 24.15 & 15.48 & 7.62 & 9.14 & 9.70 & 8.82 & 10.50 & 7.45 & 9.40 & 9.12 \\
\hline PSB & & 4.25 & 3.67 & 3.6 & 3.84 & 14.74 & 15.34 & 7.52 & 12.54 & 11.87 & 21.38 & 12.22 & 15.16 & 6.90 & 7.82 & 5.95 & 6.89 & 11.60 & 12.05 & 5 & 9.55 \\
\hline Rhizo & & 4.2 & 5.05 & 5.67 & 4.97 & 11.50 & 12.62 & 11.60 & 11.91 & 23.27 & 20.42 & 16.57 & 20.09 & 7.37 & 10.79 & 8.40 & 8.85 & 7 & 2.9 & 8.8 & 6.23 \\
\hline Azo & & 6.58 & 6.55 & 4.35 & 5.83 & 18.95 & 10.42 & 17.50 & 15.62 & 22.92 & 25.12 & 22.85 & 23.63 & 9.70 & 14.72 & 13 & 12.47 & 5.95 & 1.35 & 6.5 & 4.6 \\
\hline $\begin{array}{l}\text { PSB+Rhiz } \\
\text { o }\end{array}$ & & 4.85 & 2.52 & 5.96 & 4.44 & 20.23 & 25.72 & 28.43 & 24.79 & 20.42 & 29.68 & 28.67 & 26.26 & 13.92 & 12.67 & 5.35 & 10.65 & 6.25 & 6.9 & 5.5 & 6.22 \\
\hline PSB+Azo & & 3.62 & 9.51 & 4.2 & 5.78 & 11.93 & 19.30 & 23.72 & 18.32 & 9.12 & 19.85 & 23.95 & 17.64 & 4.15 & 16.65 & 9.92 & 10.24 & 5.15 & 10.15 & 6.10 & 7.13 \\
\hline Azo+Rhizo & & 5.16 & 2.87 & 10.05 & 6.03 & 12.76 & 12.15 & 15.63 & 13.51 & 33.40 & 10.35 & 31.03 & 24.92 & 5.92 & 6.30 & 6.75 & 6.32 & 6.75 & 6.75 & 5.25 & 6.25 \\
\hline $\begin{array}{l}\text { PSB+Azo+ } \\
\text { Rhizo }\end{array}$ & & 3.85 & 5.85 & 8.37 & 6.02 & 34.17 & 16.52 & 11.84 & 20.84 & 28.07 & 18.17 & 23.85 & 23.37 & 7.55 & 6.82 & 6.35 & 6.91 & 5.7 & 6.3 & 12.70 & 8.23 \\
\hline Mean & & 4.97 & 5.24 & 5.60 & & 17.04 & 16.00 & 18.07 & & 19.09 & 20.46 & 22.91 & & 7.90 & 10.61 & 8.18 & & 7.36 & 6.73 & 7.41 & \\
\hline $\operatorname{S.Em}( \pm)$ & & \multicolumn{4}{|c|}{0.341} & \multicolumn{4}{|c|}{1.74} & \multicolumn{4}{|c|}{1.566} & \multicolumn{4}{|c|}{0.443} & \multicolumn{4}{|c|}{0.770} \\
\hline $\begin{array}{l}\text { CD } 5 \% \text { for } \\
\text { H }\end{array}$ & & \multicolumn{4}{|c|}{0.168} & \multicolumn{4}{|c|}{0.381} & \multicolumn{4}{|c|}{0.361} & \multicolumn{4}{|c|}{0.192} & \multicolumn{4}{|c|}{0.253} \\
\hline B & & \multicolumn{4}{|c|}{0.275} & \multicolumn{4}{|c|}{0.622} & \multicolumn{4}{|c|}{0.590} & \multicolumn{4}{|c|}{0.313} & \multicolumn{4}{|c|}{0.413} \\
\hline HB & & \multicolumn{4}{|c|}{0.477} & \multicolumn{4}{|c|}{1.078} & \multicolumn{4}{|c|}{1.021} & \multicolumn{4}{|c|}{0.543} & \multicolumn{4}{|c|}{0.716} \\
\hline CV \% & & \multicolumn{4}{|c|}{11.08} & \multicolumn{4}{|c|}{7.75} & & 6.0 & 12 & & & 7.4 & 82 & & & 12.2 & 245 & \\
\hline
\end{tabular}

*Average of the three replication 
Table.6 Effect of biofertilizers and flumioxazin on fungi population

\begin{tabular}{|c|c|c|c|c|c|c|c|c|c|c|c|c|c|c|c|c|c|c|c|c|c|}
\hline \multicolumn{22}{|c|}{ Fungi Population } \\
\hline & \multirow{2}{*}{ 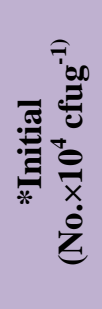 } & \multicolumn{4}{|c|}{ 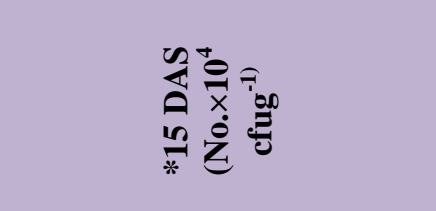 } & \multicolumn{4}{|c|}{ 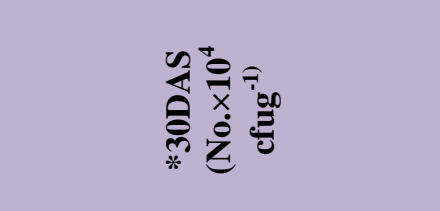 } & \multicolumn{4}{|c|}{ 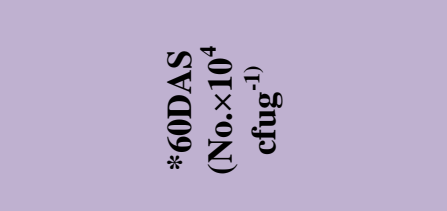 } & \multicolumn{4}{|c|}{ 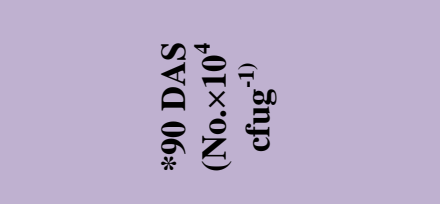 } & \multicolumn{4}{|c|}{ 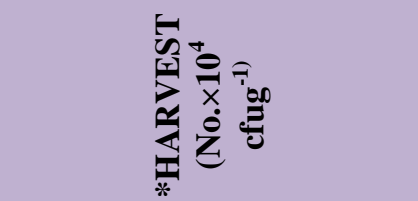 } \\
\hline $\begin{array}{l}\text { Biofert/ } \\
\text { Herbicide }\end{array}$ & & $\mathbf{H}_{\mathbf{0}}$ & $\mathbf{H}_{1}$ & $\mathbf{H}_{2}$ & Mean & $\mathbf{H}_{\mathbf{0}}$ & $\mathbf{H}_{1}$ & $\mathbf{H}_{2}$ & Mean & $\mathbf{H}_{0}$ & $\mathbf{H}_{1}$ & $\mathbf{H}_{2}$ & Mean & $\mathbf{H}_{\mathbf{0}}$ & $\mathbf{H}_{1}$ & $\mathbf{H}_{2}$ & Mean & $\mathbf{H}_{0}$ & $\mathbf{H}_{1}$ & $\mathbf{H}_{2}$ & Mean \\
\hline Control & \multirow[t]{8}{*}{16} & 6.67 & 2.67 & 10 & 6.44 & 31.52 & 2.6 & 23.30 & 19.14 & 5.33 & 3.12 & 5.25 & 4.57 & 6.5 & 4 & 4.5 & 5 & 3 & 3.5 & 11.5 & 6 \\
\hline PSB & & 3.92 & 7.33 & 7.83 & 6.36 & 46.50 & 6.8 & 22.75 & 25.35 & 3.50 & 6.87 & 9.37 & 6.58 & 3 & 6.5 & 2.5 & 4 & 18 & 6 & 1 & 8.33 \\
\hline Rhizo & & 13.25 & 6.58 & 6.50 & 8.78 & 9.10 & 14.5 & 4.40 & 9.33 & 7.12 & 6.12 & 1.87 & 5.04 & 7.5 & 20.5 & 5 & 11 & 2.5 & 2 & 9.5 & 4.67 \\
\hline Azo & & 4 & 8.58 & 5 & 5.86 & 3.4 & 16.7 & 12.65 & 10.92 & 4 & 13.5 & 4.87 & 7.46 & 5.5 & 5 & 2.67 & 4.39 & 2 & 3.5 & 5.5 & 3.67 \\
\hline PSB+Rhizo & & 7.5 & 8 & 39.5 & 18.33 & 14.65 & 10.75 & 5.80 & 10.4 & 5.62 & 3 & 5.87 & 4.83 & 5.5 & 2 & 18 & 8.5 & 9 & 1 & 15.5 & 8.5 \\
\hline PSB+Azo & & 5.25 & 3 & 5.25 & 4.5 & 3.5 & 42.9 & 10.55 & 18.98 & 4.12 & 2.62 & 4 & 3.58 & 4.5 & 16.67 & 1 & 7.39 & 4.5 & 8 & 7.5 & 6.67 \\
\hline Azo+Rhizo & & 3.75 & 6 & 6.75 & 5.5 & 16.4 & 15.13 & 5.45 & 12.33 & 1.5 & 4.37 & 11.25 & 5.71 & 1.5 & 6.5 & 16 & 8 & 6 & 4.5 & 2.5 & 4.33 \\
\hline PSB+Azo+Rhizo & & 14 & 2.25 & 6.25 & 7.5 & 13 & 13.6 & 6.95 & 11.18 & 8.75 & 36 & 1.75 & 15.5 & 11.5 & 18.33 & 3 & 10.94 & 3 & 12 & 4 & 6.33 \\
\hline Mean & & 7.29 & 5.55 & 10.88 & & 17.26 & 15.37 & 11.48 & & 4.99 & 9.45 & 5.53 & & 5.69 & 9.94 & 6.58 & & 6 & 5.06 & 7.12 & \\
\hline S.Em( $( \pm)$ & & \multicolumn{4}{|c|}{1.068} & \multicolumn{4}{|c|}{0.950} & \multicolumn{4}{|c|}{0.609} & \multicolumn{4}{|c|}{0.731} & \multicolumn{4}{|c|}{0.442} \\
\hline CD $5 \%$ for $\mathrm{H}$ & & \multicolumn{4}{|c|}{0.298} & \multicolumn{4}{|c|}{0.281} & \multicolumn{4}{|c|}{0.225} & \multicolumn{4}{|c|}{0.246} & \multicolumn{4}{|c|}{0.191} \\
\hline B & & \multicolumn{4}{|c|}{0.487} & \multicolumn{4}{|c|}{0.459} & \multicolumn{4}{|c|}{0.367} & \multicolumn{4}{|c|}{0.403} & \multicolumn{4}{|c|}{0.313} \\
\hline HB & & \multicolumn{4}{|c|}{0.843} & \multicolumn{4}{|c|}{0.795} & \multicolumn{4}{|c|}{0.637} & \multicolumn{4}{|c|}{0.698} & \multicolumn{4}{|c|}{0.542} \\
\hline CV \% & & \multicolumn{4}{|c|}{13.066} & \multicolumn{4}{|c|}{6.628} & & & & & & & & & & & & \\
\hline
\end{tabular}


Table.7 Effect of biofertilizers and flumioxazin on actinomycetes population

\begin{tabular}{|c|c|c|c|c|c|c|c|c|c|c|c|c|c|c|c|c|c|c|c|c|c|}
\hline \multicolumn{22}{|c|}{ Actinomycetes Population } \\
\hline 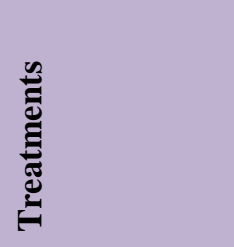 & \multirow[t]{2}{*}{ 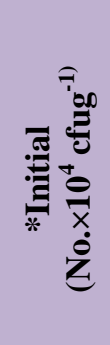 } & \multicolumn{4}{|c|}{ 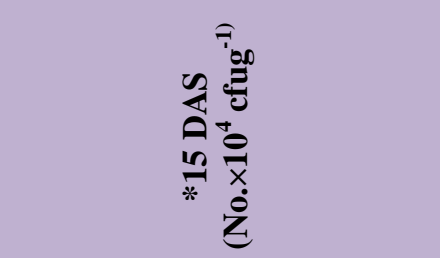 } & \multicolumn{4}{|c|}{ 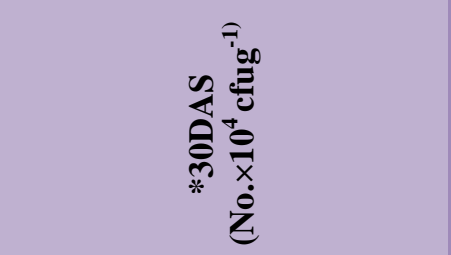 } & \multicolumn{4}{|c|}{ 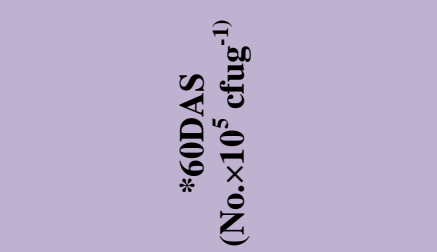 } & \multicolumn{4}{|c|}{ 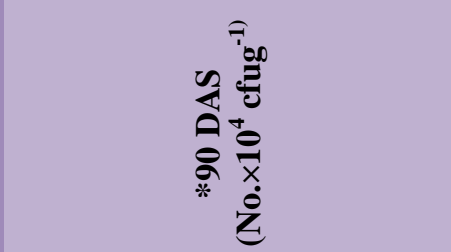 } & \multicolumn{4}{|c|}{ 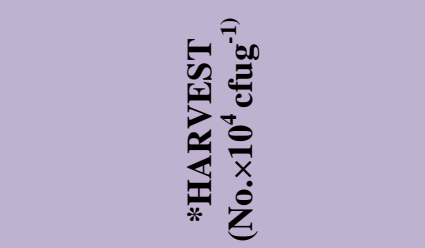 } \\
\hline $\begin{array}{l}\text { Biofert/ } \\
\text { Herbicide }\end{array}$ & & $\mathbf{H}_{\mathbf{0}}$ & $\mathbf{H}_{1}$ & $\mathbf{H}_{2}$ & Mean & $\mathbf{H}_{\mathbf{0}}$ & $\mathbf{H}_{1}$ & $\mathbf{H}_{2}$ & Mean & $\mathbf{H}_{\mathbf{0}}$ & $\mathbf{H}_{1}$ & $\mathbf{H}_{2}$ & Mean & $\mathbf{H}_{\mathbf{0}}$ & $\mathbf{H}_{1}$ & $\mathbf{H}_{2}$ & Mean & $\mathbf{H}_{\mathbf{0}}$ & $\mathbf{H}_{1}$ & $\mathbf{H}_{2}$ & Mean \\
\hline Control & \multirow[t]{8}{*}{3.0} & 8.6 & 8.25 & 9.5 & 8.78 & 33 & 28.5 & 132 & 64.5 & 0.70 & 18.70 & 3 & 7.47 & 14 & 8.5 & 15 & 12.5 & 9.5 & 7 & 10 & 8.83 \\
\hline PSB & & 7.3 & 5.45 & 8.90 & 7.22 & 59.50 & 33.50 & 35 & 42.67 & 1.20 & 1.25 & 5.80 & 2.75 & 14 & 31 & 17 & 20.67 & 6.5 & 6 & 6 & 6.17 \\
\hline Rhizo & & 9.65 & 6.75 & 8.88 & 8.43 & 37.50 & 19.50 & 34 & 30.33 & 6.05 & 11.20 & 10.60 & 9.28 & 24.5 & 25.5 & 50 & 33.33 & 3.5 & 14.5 & 8 & 8.67 \\
\hline Azo & & 12.25 & 10.05 & 10.25 & 10.85 & 74.50 & 195 & 20.50 & 96.67 & 9.60 & 1 & 2.10 & 4.23 & 10 & 11.5 & 22 & 14.5 & 2.5 & 8 & 0.5 & 3.67 \\
\hline PSB+Rhizo & & 6.5 & 50 & 14.75 & 23.75 & 51.50 & 17 & 30.50 & 33 & 3.50 & 5.65 & 0.45 & 3.2 & 2.5 & 54.5 & 8 & 21.67 & 7.5 & 3.5 & 4 & 5 \\
\hline PSB+Azo & & 9.65 & 11.20 & 17.55 & 12.8 & 33.50 & 37 & 34.50 & 35 & 9.55 & 0.25 & 0.35 & 3.38 & 12 & 5 & 39.5 & 18.83 & 8 & 2.5 & 2 & 4.17 \\
\hline Azo+Rhizo & & 9.85 & 3.60 & 49.95 & 21.13 & 96.50 & 61 & 30 & 62.5 & 1.45 & 2.65 & 0.25 & 1.45 & 28 & 14.5 & 6 & 16.17 & 1 & 8.5 & 9 & 6.17 \\
\hline $\begin{array}{l}\text { PSB+Azo+Rhi } \\
\text { zo }\end{array}$ & & 9.20 & 8.25 & 13.50 & 10.32 & 62.50 & 62 & 44.50 & 56.33 & 8.10 & 3.55 & 5 & 5.55 & 9 & 4 & 33.5 & 15.5 & 2.5 & 3 & 1 & 2.17 \\
\hline Mean & & 9.12 & 12.94 & 16.66 & & 56.06 & 56.69 & 45.12 & & 5.02 & 5.53 & 3.44 & & 14.25 & 19.31 & 23.87 & & 5.12 & 6.62 & 5.06 & \\
\hline B & & \multicolumn{4}{|c|}{0.385} & \multicolumn{4}{|c|}{1.844} & \multicolumn{4}{|c|}{0.407} & \multicolumn{4}{|c|}{0.815} & \multicolumn{4}{|c|}{0.233} \\
\hline HB & & \multicolumn{4}{|c|}{0.667} & \multicolumn{4}{|c|}{3.194} & \multicolumn{4}{|c|}{0.705} & \multicolumn{4}{|c|}{1.412} & \multicolumn{4}{|c|}{0.404} \\
\hline CV \% & & \multicolumn{4}{|c|}{6.34} & \multicolumn{4}{|c|}{7.43} & & 18 & 51 & & & 9.0 & 36 & & & & & \\
\hline
\end{tabular}

*Average of the three replication 
Table.8 Effect of biofertilizers and flumioxazin on microflora population

\begin{tabular}{|c|c|c|c|c|c|c|c|c|c|c|c|c|c|c|c|c|c|c|c|c|c|}
\hline \multicolumn{22}{|c|}{ Microflora Population } \\
\hline \multirow{2}{*}{$\begin{array}{l}\text { Biofert/ } \\
\text { Herbicide }\end{array}$} & \multirow{2}{*}{ 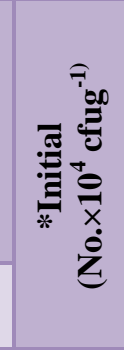 } & \multicolumn{4}{|c|}{ 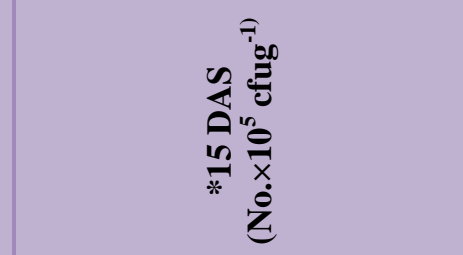 } & \multicolumn{4}{|c|}{ 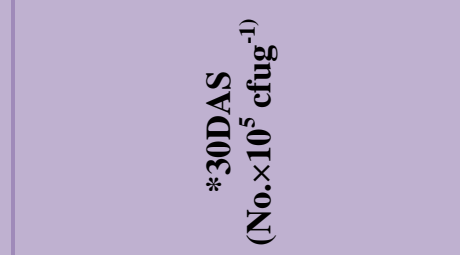 } & \multicolumn{4}{|c|}{ 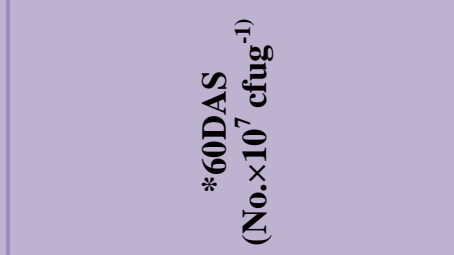 } & \multicolumn{4}{|c|}{ 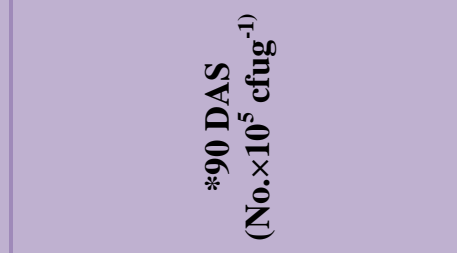 } & \multicolumn{4}{|c|}{ 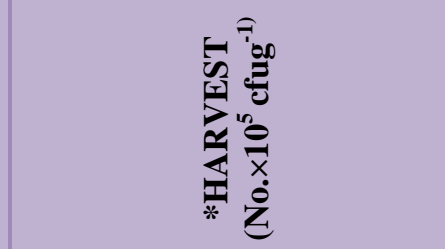 } \\
\hline & & $\mathbf{H}_{\mathbf{0}}$ & $\mathrm{H}_{1}$ & $\mathbf{H}_{2}$ & Mean & $\mathbf{H}_{\mathbf{0}}$ & $\mathbf{H}_{1}$ & $\mathbf{H}_{2}$ & Mean & $\mathbf{H}_{\mathbf{0}}$ & $\mathbf{H}_{1}$ & $\mathbf{H}_{2}$ & Mean & $\mathbf{H}_{\mathbf{0}}$ & $\mathbf{H}_{1}$ & $\mathbf{H}_{2}$ & Mean & $\mathbf{H}_{\mathbf{0}}$ & $\mathbf{H}_{1}$ & $\mathbf{H}_{2}$ & Mean \\
\hline Control & \multirow[t]{8}{*}{46.87} & 9.33 & 8.24 & 10.40 & 9.33 & 22.25 & 21.79 & 47.59 & 30.54 & 3.94 & 19.03 & 24.44 & 15.80 & 17.10 & 17.57 & 18.20 & 17.62 & 12.85 & 9.30 & 14.40 & 12.18 \\
\hline PSB & & 8.98 & 6.08 & 9.85 & 8.31 & 28.84 & 26.14 & 16.87 & 23.95 & 12.05 & 21.73 & 12.51 & 15.43 & 15.90 & 15.42 & 12.52 & 14.62 & 15.90 & 13.60 & 6.15 & 11.88 \\
\hline Rhizo & & 6.89 & 10.43 & 9.56 & 8.96 & 22.56 & 29.35 & 18.62 & 23.51 & 23.59 & 20.93 & 16.90 & 20.47 & 21.22 & 23.09 & 20.57 & 21.63 & 8.15 & 7.40 & 12.30 & 9.28 \\
\hline Azo & & 10.28 & 12.96 & 9.50 & 10.92 & 33.44 & 36.29 & 28.36 & 32.7 & 23.16 & 25.38 & 23.07 & 23.87 & 13.40 & 25.64 & 21.29 & 20.11 & 8.00 & 6.50 & 8.3 & 7.6 \\
\hline PSB+Rhizo & & 7.27 & 10.47 & 12.73 & 10.16 & 30.44 & 33.15 & 37.06 & 33.55 & 20.78 & 30.13 & 28.88 & 26.59 & 23 & 29.45 & 12.02 & 21.49 & 8.8 & 10.00 & 9.05 & 9.28 \\
\hline PSB+Azo & & 12.51 & 12.09 & 8.00 & 10.87 & 17.53 & 31.09 & 31.89 & 26.83 & 9.40 & 20.09 & 24.26 & 17.92 & 12.40 & 23.67 & 22.27 & 19.45 & 8.00 & 12.80 & 7.90 & 9.57 \\
\hline Azo+Rhizo & & 8.00 & 7.81 & 20.72 & 12.18 & 29.60 & 23.84 & 24.91 & 26.12 & 33.62 & 10.62 & 31.23 & 25.16 & 18.92 & 17.02 & 16.47 & 17.47 & 8.08 & 9.75 & 6.60 & 8.14 \\
\hline PSB+Azo+Rhizo & & 8.69 & 12.55 & 12.92 & 11.39 & 46.25 & 29.06 & 20.91 & 32.07 & 28.59 & 18.48 & 24.16 & 23.74 & 18.57 & 16.96 & 15.45 & 17.00 & 7.35 & 11.30 & 14.70 & 11.12 \\
\hline Mean & & 9.00 & 10.08 & 11.71 & & 28.86 & 28.84 & 28.28 & & 19.39 & 20.80 & 23.18 & & 17.56 & 21.10 & 17.35 & & 9.64 & 10.08 & 9.92 & \\
\hline $\operatorname{S.Em}( \pm)$ & & \multicolumn{4}{|l|}{0.902} & \multicolumn{4}{|l|}{4.571} & \multicolumn{4}{|l|}{6.542} & \multicolumn{4}{|l|}{4.335} & \multicolumn{4}{|l|}{1.241} \\
\hline CD $5 \%$ for $\mathrm{H}$ & & \multicolumn{4}{|l|}{0.274} & \multicolumn{4}{|l|}{0.617} & \multicolumn{4}{|l|}{0.738} & \multicolumn{4}{|l|}{0.601} & \multicolumn{4}{|l|}{0.321} \\
\hline B & & \multicolumn{4}{|l|}{0.447} & \multicolumn{4}{|l|}{1.007} & \multicolumn{4}{|l|}{1.205} & \multicolumn{4}{|l|}{0.981} & \multicolumn{4}{|l|}{0.525} \\
\hline HB & & \multicolumn{4}{|l|}{0.775} & \multicolumn{4}{|l|}{1.745} & \multicolumn{4}{|l|}{2.088} & \multicolumn{4}{|l|}{1.70} & \multicolumn{4}{|l|}{0.909} \\
\hline $\mathrm{CV} \%$ & & \multicolumn{4}{|l|}{9.253} & 7.459 & & & & 12.11 & & & & 11.15 & & & & 11.28 & & & \\
\hline
\end{tabular}


Fig.1 Structure and properties of flumioxazin

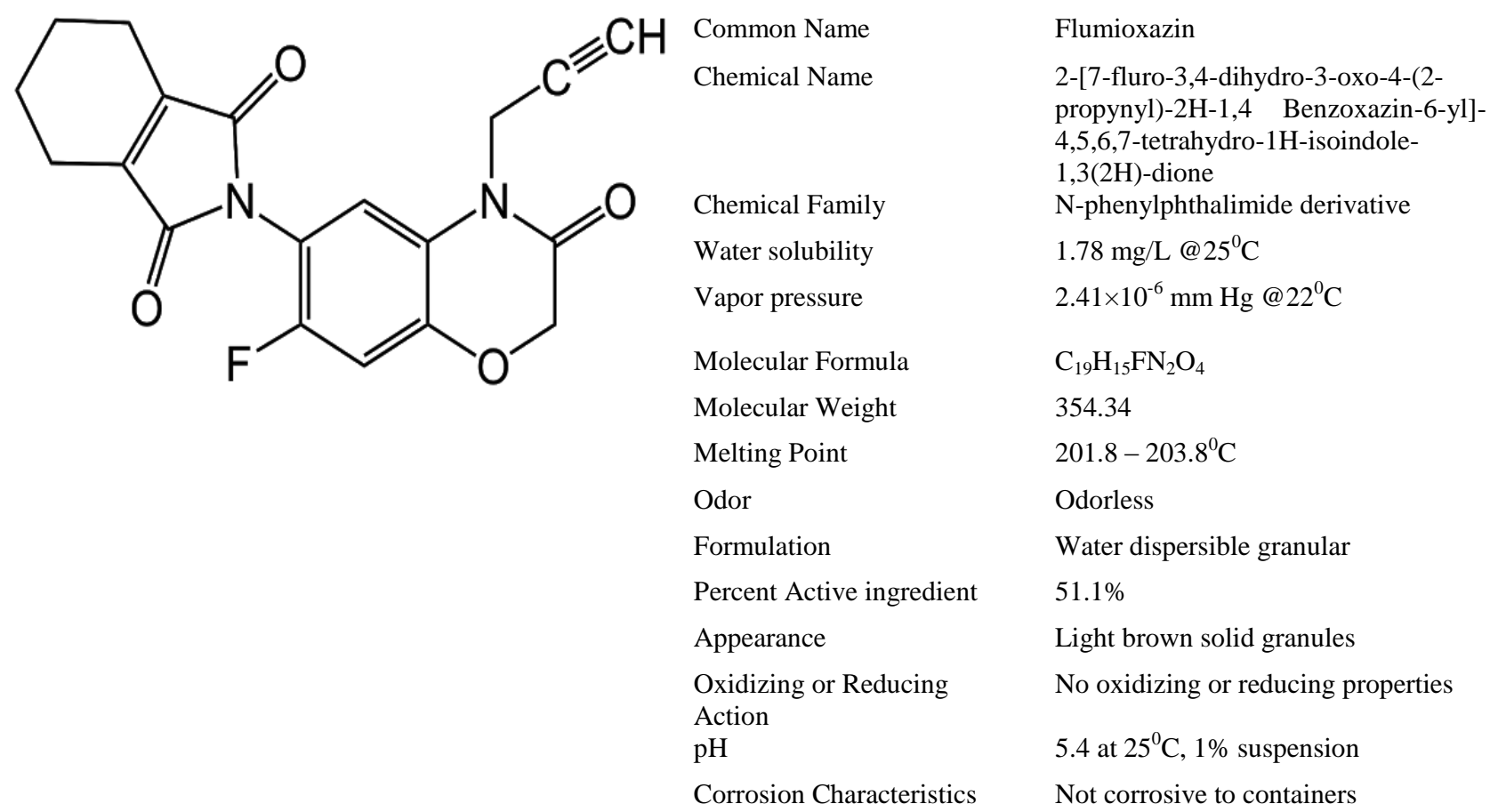

It can be concluded from the result of this experiment, that the application of herbicide Flumioxazin along with different combinations of biofertilizers PSB, Rhizobium and Azotobacter have no significant adverse effects in the physicochemical properties of soil ( $\mathrm{pH}$ and EC), available Nitrogen content, microflora population (NFB, PSB, fungi, actinomycetes) and pod yield of Groundnut. Seed inoculation with biofertilizers in combination with different doses of flumioxazin significantly increase NFB, PSB, Fungi and Actinomycetes population -78.67 to $277.33 \%,-27.03$ to $586.49 \%,-93.75$ to $12.5 \%,-83.33$ to $383.33 \%$ after harvest of groundnut, respectively as against the initial population counts and the pod yield after harvest of groundnut.

\section{References}

Dayal, Devi, Basu, M.S. and Reddy, P.S. 1987. Fertilizer use in groundnut. Technologies for better crops. Technical
Bulletin no. 30, New Delhi, Indian Council of Agricultural Research: pp. 17.

Kunc F., Tichy P. and Vancura, V. 1985. 2,4dichlorophenoxy acetic acid in the soil: Mineralization and changes in the counts of bacteria decomposers. Versailles Ed. INRA Publication. (Les Colloques de I'NRA No. 31).

Narula, N., Kumar, V., Behi, R., Deubel, A., Granse, A. and Merbach, W. 2000. Effect of P-solubilising (Azotobacter chroococcum) on $\mathrm{N}, \mathrm{P}, \mathrm{K}$ uptake in $\mathrm{P}$ responsive wheat genotypes grown under greenhouse condition. Journal of Plant Nutrition and Soil Science. 163 (4): 393-398.

Savage, G. P. and Keenan, J. I. 1994. The composition and nutritive value of groundnut kernels. In The Groundnut Crop: Scientific Basis for Improvement, pp. 173-213. Ed. J. Smart. London, UK: Chapman and Hall.

Sawicka, A and Selwet, A 1998. Effect of 
active ingredients on Rhizobium and Brady rhizobium legume dinitrogen fixation. Polish Journal of Environmental Studies. 7(5): 317- 320

Shashidhar, K.R., Narayanswamy, T.K., Bhaskar, R.N., Jagadish, B.R., Mahesh, M. and Krishra, K.S. 2009. Influence of organic nutrients on soil health and mulberry (Morus indica L.) Production. Journal of Biological Sciences. 1(1): 94100.

Sumathi, T., Janardhan, A., Srilakhmi, A., Sai Gopal, D.V.R and Narasimha, G. 2012. Impact of indigenous Microorganisms on Soil Microbial and Enzyme Activities. Archives of Applied Science Research. 4(2): 1065-1073.
Taiwo, L. B. and Oso, B. A. 1997. The influence of some pesticides on soil microbial flora in relation to changes in nutrient level, rock phosphate solubilisation and P-release under laboratory conditions. Agriculture, Ecosystem and Environment. 65(1): 5968

Usha, K., Saxena, A. and Singh, B. 2004. Rhizosphere dynamics influenced by arbuscular mycorrhizal fungus (Glomus deserticola) and related changes in leaf nutrient status and yield of Kinnow mandarin $\mathrm{x}$ Willow leaf. Australian Journal of Agricultural Research. 55(5): 571-576.

\section{How to cite this article:}

Manasi Sahoo, Soumi Mukhopadhyay and Pabitra Kumar Biswas. 2019. Effect of BioFertilizers and Flumioxazin on Microflora and Yield of Groundnut (Arachis hypogaea L.) in Alfisol of West Bengal. Int.J.Curr.Microbiol.App.Sci. 8(09): 461-473. doi: https://doi.org/10.20546/ijcmas.2019.809.056 